\title{
A General Algorithm for Dynamic Feedback Linearization of Robots with Elastic Joints
}

\author{
Alessandro De Luca Pasquale Lucibello \\ Dipartimento di Informatica e Sistemistica \\ Università degli Studi di Roma "La Sapienza" \\ Via Eudossiana 18, 00184 Roma, Italy \\ \{deluca, lucibello\}@labrob. ing. uniroma1.it
}

\begin{abstract}
For a general class of robots with elastic joints, we introduce an inversion algorithm for the synthesis of a dynamic feedback control law that gives input-output decoupling and full state linearization. Control design is performed directly on the second-order robot dynamic equations. The linearizing control law is expressed in terms of the original model components and of their time derivatives, allowing an efficient organization of computations. A tight upper bound for the dimension of the needed dynamic compensator is also obtained.
\end{abstract}

\section{Introduction}

It is well-known that the main source of vibration in industrial robot manipulators is due to the presence of joint elasticity between the driving actuators and the driven links. This is caused by the deformation of transmission elements such as harmonic drives, belts, or long shafts during high-speed motion and/or hard contact with the environment $[1,2]$. Advanced robot controllers aimed at the accurate and stable tracking of trajectories defined beyond the structural flexibility (i.e., in terms of link motion) should be designed on the basis of a more complete dynamic model of the robot [3].

The modeling of robots with elastic joints dates back to the early 80 's [4]. More recently, a detailed analysis of the model structure has been given in [5], where it was used for proving asymptotic stability of a simple regulation controller. In $[6]$, the case of motors mounted on the driven links is considered. Finally, a reduced dynamic model has been introduced in [7], neglecting gyroscopic terms in the kinetic energy of the motors.

It was shown [8] that standard tools for control- ling nonlinear systems, such as feedback linearization, input-output decoupling, or inversion control (see, e.g., [9]), are not sufficient in the case of robots with elastic joints. As a matter of fact, the complete dynamic model of robots with elastic joints fails to satisfy the necessary conditions for input-output decoupling and/or full linearization by static state feedback [3] as opposed to the case of rigid robots where the above methods are all equivalent to the 'computed torque' technique. Instead, the reduced model of elastic joint robots [7] can always be linearized and decoupled by means of a suitable nonlinear feedback from the robot state.

For the general case, it is useful to resort to the larger class of dynamic state feedback controllers. In this control scheme, the input torque applied to the robot depends both on the robot state and on the state of a dynamic compensator of proper dimension. The first linearizing and decoupling solutions were obtained for a planar $2 R$ arm (compensator of dimension four) [10] and an elbow-type $3 R$ robot (compensator of dimension six) [11]. Later on, taking advantage of the model analysis given in [5], it was proven that the whole class of robots with elastic joints can be linearized via dynamic feedback [12]. The relevance of this result for accurate trajectory tracking is that we can then easily design a global stabilizing feedback on the linear side of the problem.

On the other hand, the high complexity and the need to derive the actual controller on a case-by-case basis are the main limitations of the dynamic linearization approach. In addition, there is no general result on the dimension of the linearizing dynamic compensator which, even for a given number of elastic joints, still depends on the specific kinematic arrangement of the arm [13]. These difficulties stem from the use of nonlinear control methods based on a statespace representation of the plant. For mechanical sys- 
tems, the transformation from second-order (EulerLagrange) equations to state-space format, though simple, may obscure some relevant model property. Moreover, subsequent computations (essentially, Lie derivatives and brackets of vector fields [9]) would mix the contributions of the single dynamic terms, resulting in complicated expressions that are difficult to manipulate and debug, and also hard to be interpreted physically. For these reasons, after about ten years from the first theoretical results, there is still no implementation of a dynamic feedback linearization controller, not even for simple robots with elastic joints.

In this paper, we present a general algorithm for the design of the dynamic control law which is constructive and does not resort to state-space equations. The algorithm proceeds by solving three successive partial linearization and input-output decoupling problems, directly working with the robot dynamic model. The final control law is defined in terms of the original model components and of their time derivatives, allowing a simple and recursive organization of the required computations. Furthermore, we are able to characterize in a precise way a tight upper bound for the dimension of the dynamic compensator.

In the following analysis, we consider a class of dynamic models that, though not the most general, includes all instances considered up to now in the literature. Elastic joint robots within this class cannot be linearized nor input-output decoupled using only static state feedback. After introducing the algorithm for a generic $N$-jointed robot, we illustrate the results on a planar $2 R$ robot.

\section{Review of Dynamic Modeling}

Consider an open kinematic chain of $N+1$ rigid bodies, interconnected by $N$ joints undergoing elastic deformation. The robot is actuated by electrical drives which are assumed to be located at the joints. Let $q \in \mathbb{R}^{N}$ be the link positions, $\theta_{m} \in \mathbb{R}^{N}$ be the motor (i.e., rotor) positions, and $\theta \in \mathbb{R}^{N}$ be the motor positions as reflected through the gear ratios. We have then $\dot{\theta}_{m i}=\dot{\theta}_{i} r_{i},(i=1, \ldots, N)$, with gear ratios $r_{i} \geq 1$. Variables $q$ and $\theta$ will be used as generalized coordinates. Note that the direct kinematics of the robot arm is a function of $q$ alone. The following assumptions are made:

Assumption 1 Joint deformations are small, so that elasticity in the joint is modeled as a (undamped) linear spring.

Assumption 2 The rotors of the motors are modeled as uniform bodies having their center of mass on the rotation axis.
Assumption 2 implies that both the inertia matrix and the gravity term in the dynamic model will be independent from the position $\theta$ of the motors.

Following the Lagrangian approach, we compute the kinetic energy of the robot structure (including links and motors as rigid bodies) as

$$
T=\frac{1}{2}\left[\begin{array}{ll}
\dot{q}^{T} & \dot{\theta}^{T}
\end{array}\right]\left[\begin{array}{cc}
B(q) & S(q) \\
S^{T}(q) & J
\end{array}\right]\left[\begin{array}{c}
\dot{q} \\
\dot{\theta}
\end{array}\right],
$$

where all blocks of the inertia matrix are $N \times N$ matrices: $B(q)$ contains the inertial properties of the rigid links, $S(q)$ accounts for the inertial couplings between motors and links, while $J=\operatorname{diag}\left\{J_{1}, \ldots, J_{n}\right\}$, $J_{i}=J_{m i} r_{i}^{2}>0$, is the matrix of the effective motor inertias, being $J_{m i}$ the axial inertia of the $i$ th rotor.

Consider the standard case in which the $i$ th motor is mounted on link $i-1$ and moves link $i$. Since the kinetic energy of the $i$ th motor does not depend on the motion of the $i$ th link and of the subsequent ones, we have the following strong model property [5]:

Property 1 Matrix $S(q)$ has the upper triangular structure

$$
\left[\begin{array}{ccccc}
0 & S_{12}\left(q_{1}\right) & S_{13}\left(q_{1}, q_{2}\right) & \ldots & S_{1 N}\left(q_{1}, \ldots, q_{N-1}\right) \\
0 & 0 & S_{23}\left(q_{2}\right) & \ldots & S_{2 N}\left(q_{2}, \ldots, q_{N-1}\right) \\
\vdots & \vdots & \vdots & \ddots & \vdots \\
0 & 0 & 0 & \cdots & S_{N-1, N}\left(q_{N-1}\right) \\
0 & 0 & 0 & \cdots & 0
\end{array}\right]
$$

where the most general cascade dependence is shown for each single term.

For the ease of presentation, we will focus on a particular situation:

Assumption 3 Matrix $S$ in eq. (1) is constant.

For instance, Assumption 3 is valid for a spatial $3 R$ elbow manipulator and for planar robots with any number of rotational joints. In the latter case, it can be shown that the elements of $S$ are $S_{i j}=J_{m j} r_{j}$, apart from those entries that are structurally zero.

The potential energy is given by the sum of the gravitational energy, for both motors and links, and of the elastic energy stored at the joints. By virtue of Assumptions 1 and 2, we have

$$
U=U_{g}(q)+\frac{1}{2}(q-\theta)^{T} K(q-\theta),
$$

in which $K=\operatorname{diag}\left\{K_{1}, \ldots, K_{n}\right\}, K_{i}>0$ being the elastic constant of joint $i$.

The robot dynamic model is obtained from the Euler-Lagrange equations for the Lagrangian $L=$ 
$T-U$. Under the above assumptions, the $2 N$ secondorder differential equations have the form (see, e.g., [3] for a detailed derivation)

$$
\begin{aligned}
B(q) \ddot{q}+S \ddot{\theta}+c(q, \dot{q})+g(q)+K(q-\theta) & =0(3) \\
S^{T} \ddot{q}+J \ddot{\theta}+K(\theta-q) & =\tau,(4)
\end{aligned}
$$

where $c(q, \dot{q})$ are Coriolis and centrifugal terms, $g(q)=$ $\left(\partial U_{g} / \partial q\right)^{T}$ are gravity terms, and $\tau \in \mathbb{R}^{N}$ are the torques supplied by the motors.

We note explicitly that in the case of a single link and for some other special kinematic structures with elastic joints (e.g., a $2 R$ polar robot) it is found that $S=0$, implying no inertial couplings between the link and the motor dynamics. The same situation is forced by the modeling assumption introduced in [7], namely by considering in the angular part of the kinetic energy of each rotor only the term due to its relative rotation. When $S=0$, the model is always feedback linearizable by static state feedback. Thus, from now on we assume $S \neq 0$.

\section{Inversion Algorithm}

A dynamic state feedback control for the input torques $\tau$ in eq. (4) is a law of the form

$$
\begin{aligned}
\tau & =\alpha(x, \xi)+\beta(x, \xi) v \\
\dot{\xi} & =\gamma(x, \xi)+\delta(x, \xi) v
\end{aligned}
$$

where $x=(q, \theta, \dot{q}, \dot{\theta}) \in \mathbb{R}^{4 N}$ is the state of the robot, $\xi \in \mathbb{R}^{M}$ is the state of the dynamic compensator (of order $M$ to be defined), and $v \in \mathbb{R}^{N}$ is the new control input.

Our objective is to design such a control law so that the closed-loop system made by eqs. (3-4) and (56 ) is represented by decoupled chains of input-output integrators, i.e.,

$$
\frac{d^{\rho_{i}} q_{i}}{d t^{p_{i}}}=v_{i}, \quad i=1, \ldots, N
$$

with the additional requirement that

$$
\sum_{i=1}^{N} \rho_{i}=4 N+M
$$

where $\rho_{i}$ the closed-loop relative degree of the output variable $q_{i}$. Condition ( 8 ) implies, beside input-output decoupling, also full state linearization (in the proper coordinates) of the closed-loop system. Satisfaction of these control objectives enables to solve the problem of tracking a desired trajectory $q_{d}(t)$, in a global sense and with a prescribed linear error dynamics.
In the following, we propose a design algorithm that proceeds incrementally, first achieving partial linearization and input-output decoupling for two simpler subproblems and then obtaining from there the final solution. The main aspect is that all derivations are based directly on the robot dynamic model (3-4).

\subsection{Input-output decoupling w.r.t. $\theta$}

From the structure of eq. (4), we define the following control law for $\tau$ :

$$
\tau=J u+S^{T} \ddot{q}+K(\theta-q),
$$

where $u \in \mathbb{R}^{N}$ is a new control input. This control law imposes the dynamics

$$
\begin{aligned}
B(q) \ddot{q}+S u+c(q, \dot{q})+g(q)+K(q-\theta) & =0 \\
\ddot{\theta} & =u .
\end{aligned}
$$

The implementation of control law (9) by state feedback requires to eliminate the link acceleration $\ddot{q}$. Solving for $\ddot{q}$ in eq. (10) and substituting in eq. (9) gives

$$
\begin{aligned}
\tau= & {\left[J-S^{T} B^{-1}(q) S\right] u+K(\theta-q) } \\
& -S^{T} B^{-1}(q)[c(q, \dot{q})+g(q)+K(q-\theta)] .
\end{aligned}
$$

Equation (11) shows that a linear and decoupled relation has been obtained between each input component $u_{i}$ and each output $\theta_{i}(i=1, \ldots, N)$, by using a static state feedback law $\tau=\tau(q, \theta, \dot{q}, u)$. In the closed-loop system, we have $2 N$ states (namely, $q$ and $\dot{q}$ ) that are unobservable from the output $\theta$.

\section{$3.2 \quad$ Input-output decoupling w.r.t. $f$}

By defining a new output $f$ as

$$
f=B(q) \ddot{q}+c(q, \dot{q})+g(q)+K q,
$$

eq. (3) can be rewritten as

$$
f(q, \dot{q}, \ddot{q})+S u-K \theta=0
$$

where eq. (11) has been used. We note that output $f$ has the dimension of a generalized force. Differentiating twice eq. (14), we obtain

$$
\ddot{f}(q, \dot{q}, \ddot{q})+S \ddot{u}-K u=0 .
$$

By defining the following control law for $u$

$$
u=K^{-1}\left[S \ddot{u}+w^{\prime}\right],
$$

where $w^{\prime} \in \mathbb{R}^{N}$ is a new control input, we would simply get

$$
\ddot{f}(q, \dot{q}, \ddot{q})=w^{\prime}
$$


i.e., a linear and decoupled relation between each input $w_{i}^{\prime}$ and each output $f_{i}(i=1, \ldots, N)$. Owing to Property 1 , the control law (15) inherits a hierarchical structure and is thus well defined, even if its implementation requires input differentiation.

To avoid input differentiation, we proceed in a different way by adding on each input channel $u_{i}$ a string of integrators. In particular, $2(i-1)$ integrators, with states $\phi_{i j}$, are put on the $i$ th channel $(i=1, \ldots, N$; $j=1, \ldots, 2(i-1))$ :

$$
\begin{aligned}
u_{i} & =\phi_{i 1} \\
\dot{\phi}_{i 1} & =\phi_{i 2} \\
& \vdots \\
\dot{\phi}_{i, 2(i-1)} & =\bar{w}_{i},
\end{aligned}
$$

where $\bar{w} \in \mathbb{R}^{N}$ is a temporary control input. The total number of added integrators is $N(N-1)$. Denote by $\phi$ the vector collecting the states of all these integrators. Differentiating $2 i$ times the $i$ th scalar equation in (14), and keeping into account the dynamic extension (17), we obtain, for $i=1, \ldots, N$,

$$
\begin{aligned}
\frac{d^{2 i} f_{i}}{d t^{2 i}} & =-\sum_{j=i+1}^{N} S_{i j} \frac{d^{2 i} u_{j}}{d t^{2 i}}+K_{i} \frac{d^{2(i-1)} u_{i}}{d t^{2(i-1)}} \\
& =-S_{i, i+1} \bar{w}_{i+1}-\sum_{j=i+2}^{N} S_{i j} \phi_{j, 2 i+1}+K_{i} \bar{w}_{i} .
\end{aligned}
$$

By defining recursively the control law for $\bar{w}$

$$
\begin{aligned}
K_{N} \bar{w}_{N}= & w_{N} \\
K_{N-1} \bar{w}_{N-1}= & S_{N-1, N} \bar{w}_{N}+w_{N-1} \\
K_{i} \bar{w}_{i}= & S_{i, i+1} \bar{w}_{i+1}+\sum_{j=i+2}^{N} S_{i j} \phi_{j, 2 i+1}+w_{i} \\
& (i=N-2, N-3, \ldots, 1)
\end{aligned}
$$

we get

$$
\frac{d^{2 i} f_{i}}{d t^{2 i}}=w_{i}, \quad i=1, \ldots, N .
$$

Equations (19) show again a linear and decoupled relation between each input $w_{i}$ and each output $f_{i}$, resulting now from the application of the linear $d y-$ namic compensator $u=u(\phi, w)$ obtained through eqs. (17) and (18). Indeed, when combining this compensator with eq. (12), a nonlinear dynamic state feedback $\tau=\tau(q, \theta, \dot{q}, \phi, w)$ is defined for the original robot torque input. The total number of states of the robot and of the compensator is $N(N+3)$ whereas, from eqs. (19), the number of states on the input-output channels is $N(N+1)$. Therefore, in the closed-loop system, we have still $2 N$ unobservable states from the output $f$.

\subsection{Input-output decoupling w.r.t. $q$}

As the last algorithmic step, we tackle the inputoutput decoupling and linearization problem for the original output $q$. The mapping from $f$ to $q$, represented by eq. (13), contains the main nonlinearities of the robot link dynamics. In order to cancel them in a well-defined way, we need to dynamically balance the input-output relations in eqs. (19). In fact, differentiating $2(N-i)$ times the $i$ th equation in (19) we get

$$
\begin{aligned}
& \frac{d^{2(N-i)}}{d t^{2(N-i)}} \frac{d^{2 i} f_{i}}{d t^{2 i}}=\frac{d^{2(N-i)} w_{i}}{d t^{2(N-i)}} \\
& \quad=\frac{d^{2 N}}{d t^{2 N}}\left(b_{i}^{T}(q) \ddot{q}+c_{i}(q, \dot{q})+g_{i}(q)+K_{i} q_{i}\right)
\end{aligned}
$$

for $i=1, \ldots, N$, where $b_{i}(q)$ the $i$ th column of the link inertia matrix $B(q)$.

To avoid differentiation of the input $w$, we add $2(N-i)$ integrators, with states $\psi_{i j}$, on the $i$ th channel $(i=1, \ldots, N ; j=1, \ldots, 2(N-i))$ :

$$
\begin{aligned}
w_{i} & =\psi_{i 1} \\
\dot{\psi}_{i 1} & =\psi_{i 2} \\
& \vdots \\
\dot{\psi}_{i, 2(N-i)} & =\bar{v}_{i}
\end{aligned}
$$

where $\bar{v} \in \mathbb{R}^{N}$ is a temporary control input. The total number of integrators is again $N(N-1)$. Denote by $\psi$ the vector collecting the states of all these integrators.

Resume the vector notation and rewrite eqs. (20), using eqs. (21), as

$$
\frac{d^{2 N}}{d t^{2 N}}(B(q) \ddot{q}+c(q, \dot{q})+g(q)+K q)=\bar{v} .
$$

Performing differentation term by term gives

$$
B(q) q^{\{2(N+1)\}}+n\left(q, \dot{q}, \ldots, q^{\{2 N+1\}}\right)=\bar{v},
$$

where

$$
\begin{aligned}
n= & \sum_{k=1}^{2 N}\left(\begin{array}{c}
2 N \\
k
\end{array}\right) B^{\{k\}}(q) q^{\{2(N+1)-k\}} \\
& +c^{\{2 N\}}(q, \dot{q})+g^{\{2 N\}}(q)+K q^{\{2 N\}}
\end{aligned}
$$

and we have used the notation $a^{\{i\}}:=d^{i} a / d t^{i}$. Therefore, by defining the linearizing control law

$$
\bar{v}=B(q) v+n\left(q, \dot{q}, \ldots, q^{\{2 N+1\}}\right),
$$


we finally obtain

$$
\frac{d^{2(N+1)} q_{i}}{d t^{2(N+1)}}=v_{i}, \quad i=1, \ldots, N
$$

Note that eq. (23) can be seen a generalization of the computed torque method for rigid robots and is globally defined thanks to the positive definiteness of the link inertia matrix $B(q)$.

Input-output decoupling and linearization has been achieved by means of the nonlinear dynamic feedback $w=w\left(q, \dot{q}, \ldots, q^{\{2 N+1\}}, \psi, v\right)$, obtained from eqs. (21) and (23). Note that the dependence of this control law on $\ddot{q}$ and on higher derivatives can be eliminated recursively, in terms of the robot state $(q, \theta, \dot{q}, \dot{\theta})$ and compensator state $(\phi, \psi)$.

Define the total state of the dynamic compensator as $\xi=(\phi, \psi)$, which is of dimension $M=2 N(N-1)$. By combining eqs. (12), (17), (18), (21), and (23), we obtain a nonlinear dynamic state feedback control law $\tau=\tau(x, \xi, v)$ with the structure (5-6). Furthermore, eqs. (24) are in the form (7) with uniform relative degrees $\rho_{i}=2(N+1)$, for all $i=1, \ldots, N$. Condition $(8)$ is then fulfilled since the number of states on the input-output channels $(2 N(N+1))$ equals the sum of the number of states of the robot $(4 N)$ and of the compensator $(2 N(N-1))$. Thus, we have no more unobservable states left in the closed-loop system, which is in turn completely described by the linear inputoutput dynamics (24).

\subsection{Remarks}

- Stable tracking of a reference trajectory $q_{d}(t)$ is realized by any standard control technique for linear single input-single output systems. For example, using pole assignment we choose, for $i=1, \ldots, N$,

$$
v_{i}=q_{d i}^{\{2(N+1)\}}+\sum_{j=0}^{2 N+1} k_{i j}\left(q_{d i}^{\{i\}}-q_{i}^{\{i\}}\right),
$$

where the $k_{i j}$ 's are coefficients of Hurwitz polynomials having prescribed roots in the complex lefthalf plane. From eqs. (25), it follows that perfect tracking is obtained for $2(N+1)$-times differentiable trajectories.

- The main computational effort of the dynamic controller is concentrated in the evaluation of the term (22), which in turn requires the explicit expressions of the linearizing coordinates $q^{\{i\}}(i=$ $2, \ldots, 2 N+1$ ) (see also eqs. (25)). These computations can be customized for a specific robot arm since all components of the control law are defined in terms of the available dynamic model elements.
Also, efficiency is obtained by exploiting recurrent calculations.

- We have implicitly assumed that all the strictly upper triangular elements of matrix $S$ in eq. (3) are different from zero. If some of these elements vanish, the dimension of the required dynamic compensator may decrease together with the lengths of the input-output integrators chains (7). The output relative degrees can also become one different from the other. Therefore, the value $M=2 N(N-1)$ is in general an upper bound to the dimension of the linearizing dynamic controller.

- It is immediate to verify that, when $S=0$, the three steps of the above algorithm build up the static feedback linearizing controller of [7]. In particular, the dynamic extensions in eqs. (17) and (21) vanish.

\section{Application to a $2 \mathbf{R}$ planar robot}

The dynamic model of a two-link $(N=2)$ planar robot with elastic joints (see Fig. 1) has the form of eqs. (3-4) with

$$
\begin{aligned}
B(q) & =\left[\begin{array}{cc}
a_{1}+2 a_{3} c_{2} & a_{2}+a_{3} c_{2} \\
s y m m & a_{2}
\end{array}\right] \\
S & =\left[\begin{array}{cc}
0 & J_{m 2} r_{2} \\
0 & 0
\end{array}\right] \\
J & =\left[\begin{array}{cc}
J_{m 1} r_{1}^{2} & 0 \\
0 & J_{m 2} r_{2}^{2}
\end{array}\right] \\
c(q, \dot{q}) & =\left[\begin{array}{c}
-a_{3} s_{2}\left(\dot{q}_{2}^{2}+2 \dot{q}_{1} \dot{q}_{2}\right) \\
a_{3} s_{2} \dot{q}_{1}^{2}
\end{array}\right] \\
g(q) & =0,
\end{aligned}
$$

where $c_{2}=\cos q_{2}, s_{2}=\sin q_{2}$, and the dynamic coeffcients are

$$
\begin{aligned}
& a_{1}=I_{1}+m_{1} d_{1}^{2}+\left(m_{r 2}+m_{2}\right) \ell_{1}^{2}+J_{m 2}+I_{2}+m_{2} d_{2}^{2} \\
& a_{2}=I_{2}+m_{2} d_{2}^{2} \\
& a_{3}=m_{2} \ell_{1} d_{2},
\end{aligned}
$$

being $m_{i}$ the total mass of the $i$ th link (including, for the first link, the stator mass of the second motor), $I_{i}$ the inertia around its center of mass, $d_{i}$ the distance of the center of mass of the $i$ th link from the $i$ th joint, $\ell_{1}$ the length of the first link, and $m_{r 2}$ the mass of the rotor of the second motor.

In this case, the single nontrivial element in matrix $S$ is non-zero and the linearizing dynamic compensator has dimension $M=4$, attaining its theoretical upper 
bound. Also, from eq. (24), the closed-loop system will be equivalent to two chains of 6 input-output integrators. These results are consistent with those found in [10]. The following derivation should be compared with the (partial) results contained in that paper, in order to fully appreciate the reduction in complexity of the controller.

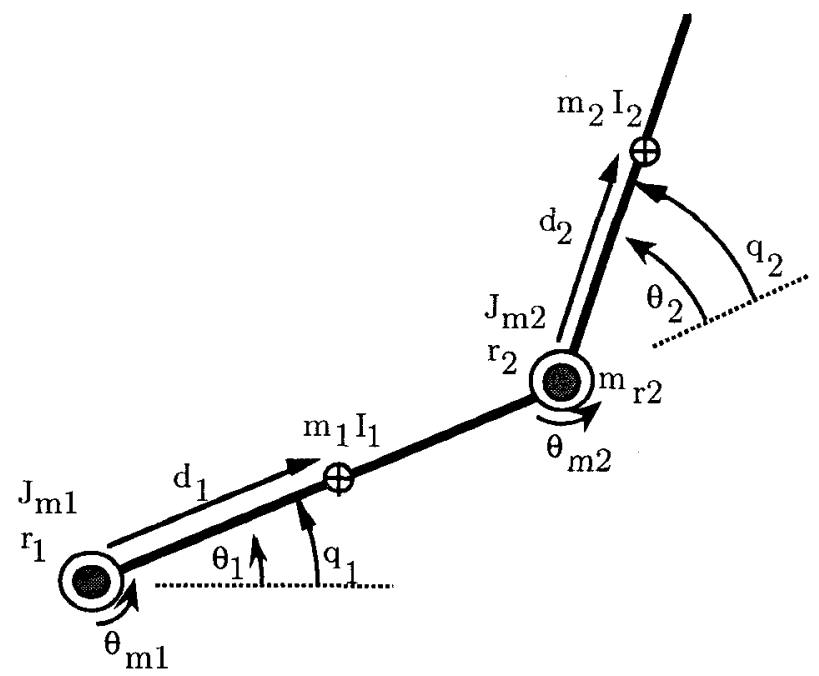

Figure 1: A 2R planar robot with elastic joints

The control law (12) becomes in this case

$$
\begin{aligned}
\tau_{1}= & J_{m 1} r_{1}^{2} u_{1}+K_{1}\left(\theta_{1}-q_{1}\right) \\
\tau_{2}= & \frac{J_{m 2} r_{2}}{\Delta}\left[r_{2} \hat{\Delta} u_{2}\right. \\
& +a_{2} K_{1}\left(q_{1}-\theta_{1}\right)-\left(a_{2}+a_{3} c_{2}\right) K_{2}\left(q_{2}-\theta_{2}\right) \\
& \left.-a_{3}^{2} s_{2} c_{2} \dot{q}_{1}^{2}-a_{2} a_{3} s_{2}\left(\dot{q}_{1}+\dot{q}_{2}\right)^{2}\right]+K_{2}\left(\theta_{2}-q_{2}\right),
\end{aligned}
$$

with $\Delta=\operatorname{det} B=a_{2}\left(a_{1}-a_{2}\right)-a_{3}^{2} \cos ^{2} q_{2}>0$, and similarly $\hat{\Delta}$ with $\hat{a}_{1}=a_{1}-J_{m 2}$ in place of $a_{1}$.

The dynamic extensions (17) and (21) are both of dimension 2. Combining them with control law (18) leads to

$$
\begin{aligned}
u_{1} & =\frac{1}{K_{1}}\left[\psi_{11}+\frac{J_{m 2} r_{2}}{K_{2}} \bar{v}_{2}\right] \\
u_{2} & =\phi_{21} \\
\dot{\phi}_{21} & =\phi_{22} \\
\dot{\phi}_{22} & =\frac{1}{K_{2}} \bar{v}_{2} \\
\dot{\psi}_{11} & =\psi_{12} \\
\dot{\psi}_{12} & =\bar{v}_{1} .
\end{aligned}
$$

Finally, the control law (23) is

$$
\begin{aligned}
& \bar{v}_{1}=\left(a_{1}+2 a_{3} c_{2}\right) v_{1}+\left(a_{2}+a_{3} c_{2}\right) v_{2}+n_{1} \\
& \bar{v}_{2}=\left(a_{2}+a_{3} c_{2}\right) v_{1}+a_{2} v_{2}+n_{2} .
\end{aligned}
$$

The two-dimensional vector $n$ is here, dropping dependencies,

$n=\dot{B} q^{\{5\}}+6 \ddot{B} q^{\{4\}}+4 B^{\{3\}} q^{\{3\}}+B^{\{4\}} \ddot{q}+c^{\{4\}}+K q^{\{4\}}$.

To get an idea of the involved computations, we give hereafter only the expression of the sum of the terms related to the link inertia matrix (first four above):

$$
\begin{aligned}
& {\left[\begin{array}{cc}
-2 a_{3} s_{2} & -a_{3} s_{2} \\
-a_{3} s_{2} & 0
\end{array}\right]\left[\begin{array}{c}
4 \dot{q}_{2} q_{1}^{\{5\}}+6 \ddot{q}_{2} q_{1}^{\{4\}} \\
+4 q_{2}^{\{3\}} q_{1}^{\{3\}}+q_{2}^{\{4\}} \ddot{q}_{1} \\
4 \dot{q}_{2} q_{2}^{\{5\}}+7 \ddot{q}_{2} q_{2}^{\{4\}} \\
+4\left(q_{2}^{\{3\}}\right)^{2}
\end{array}\right]} \\
& +\left[\begin{array}{cc}
-2 a_{3} c_{2} & -a_{3} c_{2} \\
-a_{3} c_{2} & 0
\end{array}\right]\left[\begin{array}{c}
6 \dot{q}_{2}^{2} q_{1}^{\{4\}}+12 \dot{q}_{2} \ddot{q}_{2} q_{1}^{\{3\}} \\
+\left(3 \ddot{q}_{2}^{2}+4 \dot{q}_{2} q_{2}^{\{3\}}\right) \ddot{q}_{1} \\
6 \dot{q}_{2}^{2} q_{2}^{\{4\}}+12 \dot{q}_{2} \ddot{q}_{2} q_{2}^{\{3\}} \\
+\left(3 \ddot{q}_{2}^{2}+4 \dot{q}_{2} q_{2}^{\{3\}}\right) \ddot{q}_{2}
\end{array}\right] \\
& +\left[\begin{array}{cc}
2 a_{3} s_{2} & a_{3} s_{2} \\
a_{3} s_{2} & 0
\end{array}\right]\left[\begin{array}{c}
4 \dot{q}_{2}^{3} q_{1}^{\{3\}}+6 \dot{q}_{2}^{2} \ddot{q}_{2} \ddot{q}_{1} \\
4 \dot{q}_{2}^{3} q_{2}^{\{3\}}+6 \dot{q}_{2}^{2} \ddot{q}_{2}^{2}
\end{array}\right] \\
& +\left[\begin{array}{cc}
2 a_{3} c_{2} & a_{3} c_{2} \\
a_{3} c_{2} & 0
\end{array}\right]\left[\begin{array}{c}
\dot{q}_{2}^{4} \ddot{q}_{1} \\
\dot{q}_{2}^{4} \ddot{q}_{2}
\end{array}\right] .
\end{aligned}
$$

Indeed, evaluation of terms could be organized differently. Similar formulas are obtained for $c^{\{4\}}(q, \dot{q})$.

To complete the construction of the controller, we need the explicit expressions of the linearizing coordinates. These involve computing, in the proper order, the following time derivatives as functions of the robot and compensator states:

$$
\begin{aligned}
\ddot{q}= & B^{-1}(q)\left\{K(\theta-q)-c(q, \dot{q})-\left[\begin{array}{c}
J_{m 2} r_{2} \phi_{21} \\
0
\end{array}\right]\right\} \\
q^{\{3\}}= & B^{-1}(q)\{K(\dot{\theta}-\dot{q})-\dot{c}(q, \dot{q})-\dot{B}(q) \ddot{q} \\
& \left.-\left[\begin{array}{c}
J_{m 2} r_{2} \phi_{22} \\
0
\end{array}\right]\right\} \\
q^{\{4\}=} & B^{-1}(q)\left\{\left[\begin{array}{c}
\psi_{11} \\
K_{2} \phi_{21}
\end{array}\right]\right. \\
& \left.-(K+\ddot{B}(q)) \ddot{q}-2 \dot{B}(q) q^{\{3\}}-\ddot{c}(q, \dot{q})\right\} \\
q^{\{5\}}= & B^{-1}(q)\left\{\left[\begin{array}{c}
\psi_{12} \\
K_{2} \phi_{22}
\end{array}\right]-B^{\{3\}}(q) \ddot{q}\right. \\
& \left.-(K+3 \ddot{B}(q)) q^{\{3\}}-3 \dot{B}(q) q^{\{4\}}-c^{\{3\}}(q, \dot{q})\right\}
\end{aligned}
$$

Note that inversion of the link inertia matrix should be performed only once. 
Using instead a dynamic linearization algorithm defined on the state-space representation of this robot, as in [10], leads to a complete expression of the control law contained in about 200 lines of high-level code, already after careful optimization of redundant computations.

\section{Conclusions}

For a class of robots with elastic joints in which static state feedback fails to achieve exact linearization and input-output decoupling, we have introduced a new general algorithm for the synthesis of a model-based dynamic state feedback law reaching the same control goal.

Only the second-order robot equations have been used in the analysis and in the control design. More physical insight is gained by working directly with the dynamic model terms, while control computations can be efficiently organized and thus quite reduced.

Although we have assumed throughout the paper ideal conditions, with perfect knowledge of the robot dynamic models and availability of full state measures, the present result can be used as a starting point for the definition of adaptive and robust controllers, possibly based only on feedback from the link position output.

We are currently considering the extensions of this algorithm to the case of robots with mixed (rigid or elastic) type of joints [14], or with visco-elastic joints. In the latter case, a zero-dynamics appears and only input-output linearization, with internal stability, can be achieved.

\section{Acknowledgements}

This work is supported by MURST $40 \%$ and $C N R$ 97.4585. CTO7 funds.

\section{References}

[1] L.M. Sweet and M.C. Good, "Redefinition of the robot motion control problem," IEEE Control Systems Mag., vol. 5, no. 3, pp. 18-24, 1985.

[2] E. Rivin, Mechanical Design of Robots, McGrawHill, New York, 1988.

[3] A. De Luca and P. Tomei, "Elastic joints," in Theory of Robot Control, C. Canudas de Wit, B. Siciliano, G. Bastin (Eds.), pp. 179-217, SpringerVerlag, Berlin, 1996.

[4] G. Cesareo, F. Nicolò, and S. Nicosia, "DYMIR: A code for generating dynamic model of robots,"
1984 IEEE Int. Conf. on Robotics and Automation, Atlanta, GA, pp. 115-120, 1984.

[5] P. Tomei, "A simple PD controller for robots with elastic joints," IEEE Trans. on Automatic Control, vol. 36, no. 10, pp. 1208-1213, 1991.

[6] S.H. Murphy, J.T. Wen, and G.N. Saridis, "Simulation and analysis of flexibly jointed manipulators," 29th IEEE Conf. on Decision and Control, Honolulu, HI, pp. 545-550, 1990.

[7] M.W. Spong, "Modeling and control of elastic joint robots," ASME J. of Dynamic Systems, Measurement, and Control, vol. 109, pp. 310-319, 1987.

[8] R. Marino and S. Nicosia, "On the feedback control of industrial robots with elastic joints: A singular perturbation approach," Rep. R-84.01, Dipartimento di Ingegneria Elettronica, Università di Roma "Tor Vergata", June 1984.

[9] A. Isidori, Nonlinear Control Systems, 3rd Ed., Springer-Verlag, Berlin, 1995.

[10] A. De Luca, "Dynamic control of robots with joint elasticity," 1988 IEEE Int. Conf. on Robotics and Automation, Philadelphia, PA, pp. 152-158, 1988 (also in Robot Control: Dynamics, Motion Planning, and Analysis, M.W. Spong, F. Lewis, C.T. Abdallah (Eds.), IEEE Press, 1992).

[11] A. De Luca, A. Isidori, and F. Nicolò, "Control of robot arm with elastic joints via nonlinear dynamic feedback," 24th IEEE Conf. on Decision and Control, Ft. Lauderdale, FL, pp. 1671-1679, 1985.

[12] A. De Luca and L. Lanari, "Robots with elastic joints are linearizable via dynamic feedback," 34th IEEE Conf. on Decision and Control New Orleans, LA, pp. 3895-3897, 1995.

[13] A. De Luca, "Control properties of robot arms with joint elasticity," in Analysis and Control of Nonlinear Systems, C.I. Byrnes, C.F. Martin, R.E. Saeks (Eds.), pp. 61-70, North-Holland, Amsterdam, 1988.

[14] A. De Luca, "Decoupling and feedback linearization of robots with mixed rigid/elastic joints," 1996 IEEE Int. Conf. on Robotics and Automation, Minneapolis, MN, pp. 816-821, 1996. 